\title{
On intersecting subgroups of Brunnian link groups
}

\author{
FENGCHUN LEI \\ JIE WU \\ YU ZHANG
}

\begin{abstract}
Let $G\left(L_{n}\right)$ be the link group of a Brunnian $n$-link $L_{n}$ and $R_{i}$ be the normal closure of the $i^{\text {th }}$ meridian in $G\left(L_{n}\right)$ for $1 \leq i \leq n$. In this article, we show that the intersecting subgroup $R_{1} \cap R_{2} \cap \cdots \cap R_{m}$ coincides with the iterated symmetric commutator subgroup $\prod_{\sigma \in \Sigma_{m}}\left[\left[R_{\sigma(1)}, R_{\sigma(2)}\right], \ldots, R_{\sigma(m)}\right]$ for $2 \leq m \leq n$ using the techniques of homotopy theory. Moreover, we give a presentation for the intersecting subgroup $R_{1} \cap R_{2} \cap \cdots \cap R_{n}$.
\end{abstract}

55Q40; 57M25

\section{Introduction}

An $n$-component link $L_{n}=\left\{l_{1}, l_{2}, \ldots, l_{n}\right\}$ is called a Brunnian link, named for Hermann Brunn [5], if it is a nontrivial link that becomes trivial when any component is removed. The simplest and best known example of Brunnian link are the Borromean rings. Milnor [25] classified Brunnian links up to link homotopy; he called them almost trivial links before Debrunner [9] named them Brunnian in 1961. The $C_{n}$-moves and the finite-type invariants have been studied by Habiro and Meilhan $[13 ; 14 ; 15 ; 16]$ and Miyazawa and Yasuhara [26]. We refer to Mangum and Stanford [23], Meilhan and Yasuhara [24] and Ozawa [27] for more results. An important application of Brunnian links to mathematical chemistry was given by Chichak et al [6].

For an $n$-link $L_{n}=\left\{l_{1}, l_{2}, \ldots, l_{n}\right\}$, we define the link group $G\left(L_{n}\right)$ as the fundamental group of the link complement $S^{3} \backslash L_{n}$. From the work of Mangum and Stanford [23], Brunnian links are determined by their link complements, so the link group $G\left(L_{n}\right)$ plays an important role in studying a Brunnian link $L_{n}$. Let $d_{i} L_{n}=\left\{l_{1}, l_{2}, \ldots, l_{i-1}, l_{i+1}, \ldots, l_{n}\right\}$ be the $(n-1)$-link obtained by deleting the $i^{\text {th }}$ component $l_{i}$ from the $n$-link $L_{n}$. There is a group homomorphism $h_{i}$ from $G\left(L_{n}\right)$ to $G\left(d_{i} L_{n}\right)$, induced by the inclusion of the link complements $S^{3} \backslash L_{n} \hookrightarrow S^{3} \backslash d_{i} L_{n}$. The kernel of $h_{i}$ is denoted by $R_{i}$. If we let $\left\langle\alpha_{i}\right\rangle$ be the normal closure of the meridian of $l_{i}$ in $G\left(L_{n}\right)$, then $\left\langle\alpha_{i}\right\rangle=R_{i}$. 
In this paper, we are interested in exploring the intersecting subgroup $R_{1} \cap R_{2} \cap \cdots \cap R_{m}$ of a Brunnian link group $G\left(L_{n}\right)$ for $2 \leq m \leq n$. Let $\beta_{i}$ be the $i^{\text {th }}$ longitude of $L_{n}$. Since $L_{n}$ is Brunnian, the homotopy class $\left[\beta_{i}\right]$ is in $R_{1} \cap \cdots \cap R_{i-1} \cap R_{i+1} \cap \cdots \cap R_{n}$ for each $i$. For some Brunnian links $L_{n}$, for instance the Whitehead link, the homotopy classes of all of the longitudes lie in the full intersecting subgroup $R_{1} \cap \cdots \cap R_{n}$.

Let $N$ and $H$ be subgroups of a group $G$. Recall that the commutator subgroup [ $N, H]$ is defined as the subgroup of $G$ generated by the commutators $[g, h]=g^{-1} h^{-1} g h$ for $g \in N$ and $h \in H$. If $N$ and $H$ are normal subgroups, then $[N, H]$ is contained in the intersecting subgroup $N \cap H$. Hence, for each permutation $\sigma \in \Sigma_{m}$, the iterated commutator subgroup

$$
\left[\left[R_{\sigma(1)}, R_{\sigma(2)}\right], \ldots, R_{\sigma(m)}\right] \leq R_{1} \cap R_{2} \cap \cdots \cap R_{m} .
$$

It follows that the symmetric commutator subgroup

$$
\left[R_{1}, R_{2}, \ldots, R_{m}\right]_{S}=\prod_{\sigma \in \Sigma_{m}}\left[\left[R_{\sigma(1)}, R_{\sigma(2)}\right], \ldots, R_{\sigma(m)}\right]
$$

is a subgroup of $R_{1} \cap R_{2} \cap \cdots \cap R_{m}$. Using a recent result of Ellis and Mikhailov [10] together with the techniques of homotopy theory, we are able to determine the intersecting subgroup $R_{1} \cap R_{2} \cap \cdots \cap R_{m}$ for $2 \leq m \leq n$.

Theorem 1.1 Suppose that $L_{n}=\left\{l_{1}, l_{2}, \ldots, l_{n}\right\}$ is an $n$-component Brunnian link and $G\left(L_{n}\right)$ is the link group of $L_{n}$. Let $R_{i}$ be the normal closure of the $i^{\text {th }}$ meridian in $G\left(L_{n}\right)$. Then

for $2 \leq m \leq n$.

$$
\bigcap_{i=1}^{m} R_{i}=\left[R_{1}, \ldots, R_{m}\right]_{S}
$$

Let $R_{1}, R_{2}, \ldots, R_{m}$ be subgroups of a group $G$. There is a Brown-Loday construction of non-commutative tensor product $T\left(R_{1}, R_{2}, \ldots, R_{m}\right)$ defined in terms of generatorrelation system [4]. The construction of $T\left(R_{1}, R_{2}, \ldots, R_{m}\right)$ will be recalled in the next section.

Our second result gives a presentation of the intersecting subgroup $R_{1} \cap \cdots \cap R_{m}$ in terms of the Brown-Loday construction.

Theorem 1.2 Given the same assumptions of Theorem 1.1, we have:

(1) There is a short exact sequence of groups

$$
\bigoplus^{n-1} \mathbb{Z} \longmapsto T\left(R_{1}, R_{2}, \ldots, R_{n}\right) \rightarrow \bigcap_{i=1}^{n} R_{i} .
$$


(2) There is a short exact sequence of groups

$$
\underset{\text { for } 2 \leq m<n .}{\bigoplus} \mathbb{Z} \succ T\left(R_{1}, R_{2}, \ldots, R_{m}\right) \rightarrow \bigcap_{i=1}^{m} R_{i}
$$

Remark The combinatorial problem of determining the intersecting subgroups is technically difficult in general, and related to the longstanding unsolved classical Whitehead asphericity question in low-dimensional topology; see Bogley [3]. The question on the determination of Brunnian braids answered in Bardakov, Mikhailov, Vershinin and $\mathrm{Wu}$ [1], Gurzo [12], Johnson [17], Levinson [18; 19] and $\mathrm{Li}$ and $\mathrm{Wu}$ [20] concerns the intersecting subgroups of braid groups with connections to homotopy theory; see Berrick, Cohen, Wong and Wu [2] and Cohen and $\mathrm{Wu}[7 ; 8]$. As a development of Brown and Loday's program [4], recent progress made by Ellis and Mikhailov gives a possibility to determine certain intersecting subgroups in terms of commutator subgroups with obstructions given by homotopy groups. It was then discovered by Fang, Lei and $\mathrm{Wu}$ [11] and $\mathrm{Li}$ and $\mathrm{Wu}$ [20] that the quotient group $\left(R_{1} \cap \cdots \cap R_{n}\right) /\left[R_{1}, \ldots, R_{n}\right]_{S}$ is isomorphic to the homotopy group $\pi_{n}\left(S^{3}\right)$ for a strongly nonsplittable $n$-link $L$, where $R_{i}$ is the normal closure of the $i^{\text {th }}$ meridian and a link is called strongly nonsplittable if any of its nonempty sublink is nonsplittable. In other words, the determination of the intersecting subgroup of strongly nonsplittable links $L$ is equally difficult to the determination of the homotopy group $\pi_{n}\left(S^{3}\right)$, even in the simplest case that $L$ is a Hopf $n$-link. Since the Brunnian links are not strongly nonsplittable, we cannot apply the results of $[11 ; 20]$ to answering the question of the intersecting subgroup of Brunnian links. However, we observe in Claim 2.2 that the connectivity hypothesis Ellis and Mikhailov [10] is satisfied for Brunnian link groups. This gives us the chance to determine the intersecting subgroup of Brunnian link groups in this article. As an application to Brunnian link groups, by Theorem 1.1 and Theorem 1.2(1) there is an exact sequence

$$
1 \rightarrow \bigoplus^{n-1} \mathbb{Z} \rightarrow T\left(R_{1}, R_{2}, \ldots, R_{n}\right) \rightarrow G\left(L_{n}\right) \rightarrow F_{n-1}^{\times n},
$$

where $F_{n-1}$ is the free group of rank $n-1$. This gives a connection between Brown and Loday's theory on non-commutative tensor products and Brunnian links.

We should point out that by Theorem 1.1, the connectivity hypothesis of Ellis and Mikhailov [10] is also satisfied for almost Brunnian link groups along the lines of the proof of Lemma 2.1, where a link is called almost Brunnian if it becomes Brunnian after removing any of its components. Of course, the full determination of the intersecting subgroup of almost Brunnian link groups will technically depend on the determination 
of the homotopy type of the homotopy colimit of a cubical diagram, as we will see in the case of Brunnian link groups in this article. The procedure can be continued for more general links until the intersecting subgroup differs from the symmetric commutator subgroup with obstruction given by the homotopy group of the homotopy colimit. This article highlights the homotopy theoretic methodology for determining the intersecting subgroup of link groups.

In the next section, we will lay out the necessary preliminaries for proving our theorems. In Section 3, we'll study the homotopy type of the space $X\left(L_{n}\right)^{m}$. The proofs of Theorems 1.1 and 1.2 will be given in Section 4.

Acknowledgements The first and the second authors were supposed by a grant of NSFC number 11329101 of China. The second author was also supported by the Academic Research Fund of the National University of Singapore R-146-000-190-112. The third author was supported by a grant of NSFC number 11101103 of China.

\section{Preliminaries}

In this section we will review some results which we need to prove our main theorems, including symmetric commutator subgroups, colimits of classifying spaces and homotopy groups of homotopy colimits.

\section{A Symmetric commutator subgroups}

Let $G$ be a group with subgroups $R_{j}$ for $1 \leq j \leq n$. The concept of the symmetric commutator subgroup of $R_{1}, R_{2}, \ldots, R_{n}$, denoted by $\left[R_{1}, R_{2}, \ldots, R_{n}\right]_{S}$, was introduced by $\mathrm{Li}$ and $\mathrm{Wu}[20]$ with the equation

$$
\left[R_{1}, R_{2}, \ldots, R_{n}\right]_{S}=\prod_{\sigma \in \Sigma_{n}}\left[\left[R_{\sigma(1)}, R_{\sigma(2)}\right], \ldots, R_{\sigma(n)}\right]
$$

where $\Sigma_{n}$ is the $n^{\text {th }}$ symmetric group and $\left[\left[R_{\sigma(1)}, R_{\sigma(2)}\right], \ldots, R_{\sigma(n)}\right]$ is the subgroup generated by the left iterated commutators $\left[\left[\left[g_{1}, g_{2}\right], g_{3}\right], \ldots, g_{n}\right]$ with $g_{i} \in R_{\sigma(i)}$.

In the same paper, the following theorem was also shown.

Theorem [20, Theorem 1.2] Let $R_{j}$ be any normal subgroup of a group $G$ with $1 \leq j \leq n$. Then

$$
\left[R_{1}, \ldots, R_{n}\right]_{S}=\prod_{\sigma \in \Sigma_{n-1}}\left[\left[R_{1}, R_{\sigma(2)}\right], \ldots, R_{\sigma(n)}\right]
$$

where $\Sigma_{n-1}$ acts on $\{2,3, \ldots, n\}$. 
A bracket arrangement of weight $n$ in a group $G$ is given by Magnus, Karrass and Solitar [22] as a map $\beta^{n}: G^{n} \rightarrow G$ defined inductively by

$$
\beta^{1}=\operatorname{id}_{G}, \quad \beta^{2}\left(g_{1}, g_{2}\right)=\left[g_{1}, g_{2}\right],
$$

where $g_{1}$ and $g_{2}$ are arbitrary elements in $G$ and $\left[g_{1}, g_{2}\right]$ is the commutator of $g_{1}$ and $g_{2}$. Inductively, suppose that the bracket arrangements of weight $k, \beta^{k}$, are defined for $k=1,2, \ldots, n-1$. Then the bracket arrangements of weight $n, \beta^{n}$, is defined by the composition

$$
G^{n}=G^{k} \times G^{n-k} \stackrel{\beta^{k} \times \beta^{n-k}}{\longrightarrow} G \times G \stackrel{\beta^{2}}{\longrightarrow} G
$$

for any $\beta^{k}$ and $\beta^{n-k}$.

Closely related to the symmetric commutator subgroup is the fat commutator subgroup. For a group $G$ with a sequence of subgroups $R_{j}, 1 \leq j \leq n$. The fat commutator subgroup $\left[\left[R_{1}, \ldots, R_{n}\right]\right]$ is defined to be the subgroup of $G$ generated by all of the commutators

$$
\beta^{t}\left(g_{i_{1}}, \ldots, g_{i_{t}}\right)
$$

where

(a) $1 \leq i_{s} \leq n$,

(b) $\left\{i_{1}, \ldots, i_{t}\right\}=\{1, \ldots, n\}$, ie each integer in $\{1,2, \ldots, n\}$ appears as at least one of the integers $i_{s}$,

(c) $g_{j} \in R_{j}$

(d) $\beta^{t}$ runs over all of the bracket arrangements of weight $t, t \geq n$.

From the definition, it is clear that the symmetric commutator subgroup is a subgroup of the fat commutator subgroup. Moreover, the following result was also shown by $\mathrm{Li}$ and $\mathrm{Wu}$ [20], and plays an important role in our proof of Theorem 1.1.

Theorem [20, Theorem 1.1] Let $R_{j}$ be any normal subgroup of a group $G$ with $1 \leq j \leq n$. Then

$$
\left[\left[R_{1}, R_{2}, \ldots, R_{n}\right]\right]=\left[R_{1}, \ldots, R_{n}\right]_{S}
$$

This theorem tells us that the fat commutator subgroup is in fact the same as the symmetric commutator subgroup.

We'll also need another result of Wu. 
Theorem [28, Corollary 3.5] Let $G=F(S)$ be a free group generated by $S$ and $F\left(T_{i}\right)$ be subgroups of $G$ freely generated by $T_{i} \subset S$ for $1 \leq i \leq k$. Let $R_{i}$ be the kernel of the projection homomorphism $\pi_{i}: G \rightarrow F\left(T_{i}\right)$. Then

$$
\bigcap_{i=1}^{k} R_{i}=\left[\left[R_{1}, R_{2}, \ldots, R_{k}\right]\right] .
$$

Commutator subgroups have many important applications, especially to homotopy groups and geometric groups. In [20], $\mathrm{Li}$ and $\mathrm{Wu}$ used the commutator subgroups to give a connection between links and homotopy groups. In the present paper, we apply the commutator subgroups to study the homotopy groups of the homotopy colimits of classifying spaces for Brunnian link groups.

\section{B Homotopy colimits of classifying spaces}

In this section, we review some facts about the colimits of classifying spaces. Our terminology here is coincident with that of Ellis and Mikhailov [10].

Roughly speaking, a classifying space $B G$ of a topological group $G$ is a topological space that is the quotient of a weakly contractible space (ie a topological space whose homotopy groups are all trivial) by a free action of $G$. The existence of a classifying space for discrete groups is a classical result. For a discrete group $G$, the classifying space $B G$ is a path-connected topological space whose fundamental group is isomorphic to $G$ and all higher homotopy groups are trivial, ie $B G$ is a $K(G, 1)$-space. Of course, for a group $G$, the classifying space is not unique. It was shown that if $X$ is a classifying space for $G$ and $Y$ is a classifying space for $K$, then $X \times Y$ is a classifying space for $G \times K$. Some of the simple examples of classifying spaces are:

(1) The circle $S^{1}$ is a classifying space for the infinite cyclic group $\mathbb{Z}$.

(2) The $n$-torus $T^{n}$ is a classifying space for the free abelian group of rank $n, \mathbb{Z}^{n}$.

(3) The wedge of $n$ circles $\bigvee^{n} S^{1}$ is a classifying space for the free group of rank $n$. Suppose that $L_{n}$ is an $n$-Brunnian link and $R_{i}$ is the normal closure of the $n^{\text {th }}$ meridian in $G\left(L_{n}\right)$. For each proper subset $I \subset\{1,2, \ldots, n\}, G\left(L_{n}\right) / \prod_{I} R_{i}$ is a free group of rank $n-|I|$, so $B\left(G\left(L_{n}\right) / \prod_{I} R_{i}\right)=\bigvee^{n-|I|} S^{1}$.

(4) It has been shown [11, Theorem 2.1] that a link in $S^{3}$ is nonsplittable if and only if the link complement is a $K(\pi, 1)$-space. So for an $n$-Brunnian link $L_{n}$ with link group $G\left(L_{n}\right), B G\left(L_{n}\right)=S^{3} \backslash L_{n}$ is a classifying space. 
We are interested in the homotopy colimits of classifying spaces. In order to have an intuitive image of homotopy colimits, we describe the homotopy push-out (colimit) of a diagram of spaces

$$
Z \stackrel{g}{\longleftarrow} X \stackrel{f}{\longrightarrow} Y .
$$

We want the homotopy push-out to preserve the homotopy type of $X$. So, we will first thicken $X$ to get $X \times[0,1]$ and then identify $X \simeq X \times\{0\}$ with $f(X) \subset Y$ and $X \simeq X \times\{1\}$ with $g(X) \subset Z$. Therefore, the homotopy push-out is just the quotient space

$$
Q=Y \amalg(X \times[0,1]) \amalg Z / \sim,
$$

where $(x, 0) \sim f(x)$ and $(x, 1) \sim g(x)$.

In a more general sense than homotopy push-out, the homotopy colimit will be a functor from the category of diagrams of spaces into the category of topological spaces $\mathscr{T}$. Suppose that $\mathscr{D}$ is a small category, ie a category where both the class of objects and class of morphisms are actually just sets. Denote by $\mathscr{T}^{\mathscr{D}}$ the category of functors $F: \mathscr{D} \rightarrow \mathscr{T}$ and natural transformations. That is, $\mathscr{T}^{\mathscr{D}}$ is the category of diagrams of spaces with the shape of $\mathscr{D}$. Our homotopy colimit is a functor

$$
\operatorname{hocolim}_{\mathscr{D}}: \mathscr{T}^{\mathscr{D}} \rightarrow \mathscr{T}
$$

which satisfies the following properties:

(1) (Homotopy invariance property) Suppose that $F, F^{\prime}: \mathscr{D} \rightarrow \mathscr{T}$ are two functors and there is a natural transformation $\tau: F \rightarrow F^{\prime}$ such that $\tau_{d}: F(d) \rightarrow F^{\prime}(d)$ is a homotopy equivalence for all $d \in \mathscr{D}$, then

$$
\operatorname{hocolim}(F) \simeq \operatorname{hocolim}\left(F^{\prime}\right)
$$

(2) If all the maps between spaces are cofibrations, then the homotopy colimit is coincident with the usual colimit, ie if $F(\alpha): F(d) \rightarrow F\left(d^{\prime}\right)$ is a cofibration for every $\alpha: d \rightarrow d^{\prime}$ in $\mathscr{D}$, then

$$
\operatorname{hocolim}(F) \simeq \operatorname{colim}(F) .
$$

What inspired us was the work on homotopy colimits given by cubical diagrams by Ellis and Mikhailov [10]. In the next section, we will review some of their results and settle down some important lemmas we need later for the proofs of our theorems. 


\section{C Homotopy groups of homotopy colimits}

Let $G$ be a group. We recall the definition of connectedness for $m$-tuples of normal subgroups $\left(R_{1}, R_{2}, \ldots, R_{m}\right)$ from [10].

Definition The $m$-tuple $\left(R_{1}, R_{2}, \ldots, R_{m}\right)$ is connected if either $m \leq 2$ or $m \geq 3$ and for all subsets $I, J \subset\{1,2, \ldots, m\}$ with $|I| \geq 2,|J| \geq 1$ we have

$$
\left(\bigcap_{i \in I} R_{i}\right) \prod_{j \in J} R_{j}=\bigcap_{i \in I}\left(R_{i} \prod_{j \in J} R_{j}\right) .
$$

In this paper, we study the homotopy colimit $X$ of the spaces $B\left(G / \prod_{i \in I} R_{i}\right)$, where $I$ ranges over all strict subsets $I \subsetneq\{1,2, \ldots, m\}$. The following theorem of Ellis and Mikhailov is important for our proofs.

Theorem [10, Theorem 1] If the $(m-1)$-tuple $\left(R_{1}, \ldots, \hat{R}_{i}, \ldots, R_{m}\right)$ is connected for each $1 \leq i \leq m$, then the homotopy colimit $X$ satisfies

$$
\begin{aligned}
\pi_{m}(X) & \cong \frac{R_{1} \cap \ldots \cap R_{m}}{\prod_{I \cup J=\{1,2, \ldots, m\}, I \cap J=\varnothing}\left[\bigcap_{i \in I} R_{i}, \bigcap_{j \in J} R_{j}\right]}, \\
\pi_{m+1}(X) & \cong \operatorname{ker}\left(\partial: T\left(R_{1}, \ldots, R_{m}\right) \rightarrow G\right) .
\end{aligned}
$$

The connectivity condition in this theorem is crucial. The following lemma ensures the connectivity property of the subgroups in our results.

Lemma 2.1 For a Brunnian link $L_{n}=\left\{l_{1}, l_{2}, \ldots, l_{n}\right\}$ as described above, the $k$-tuple $\left(R_{i_{1}}, \ldots, R_{i_{k}}\right)$ is connected for each $1 \leq k \leq n-1$ and $i_{j} \in\{1, \ldots, n\}$.

Before we prove the lemma, we make the following claim for simplicity.

Claim 2.2 Let $\pi: G \rightarrow G / \prod_{j \in J} R_{j} ; x \mapsto \bar{x}$ be the quotient map. Equation (7) in the definition of connectivity is equivalent to the equality

$$
\bigcap_{i \in I} R_{i}=\bigcap_{i \in I} \overline{R_{i}}
$$

Proof First, suppose that (10) is true, ie

$$
\overline{\bigcap_{i \in I}} R_{i}=\left(\bigcap_{i \in I} R_{i}\right) / \prod_{j \in J} R_{j}=\bigcap_{i \in I}\left(R_{i} / \prod_{j \in J} R_{j}\right)=\bigcap_{i \in I} \overline{R_{i}} .
$$


Notice that $\left(\bigcap_{i \in I} R_{i}\right)\left(\prod_{j \in J} R_{j}\right) \subseteq R_{i}\left(\prod_{j \in J} R_{j}\right)$. Therefore $\left(\bigcap_{i \in I} R_{i}\right)\left(\prod_{j \in J} R_{j}\right) \subseteq$ $\bigcap_{i \in I}\left(R_{i}\left(\prod_{j \in J} R_{j}\right)\right)$.

On the other hand, we pick any $x \in \bigcap_{i \in I}\left(R_{i}\left(\prod_{j \in J} R_{j}\right)\right)$, ie $x \in R_{i}\left(\prod_{j \in J} R_{j}\right)$ for any $i$. The image of $x, \pi(x)$, belongs to $\overline{R_{i}}$ for all $i$. So $\pi(x) \in \bigcap_{i \in I} \bar{R}_{i}=$ $\overline{\bigcap_{i \in I} R_{i}}$, where the last equality follows from (10). There exists $y \in \bigcap_{i \in I} R_{i}$ such that $\pi(y)=\pi(x)$. So $\pi\left(y^{-1} x\right)=e$, ie $y^{-1} x \in \operatorname{ker} \pi=\prod_{j \in J} R_{j}$. Then $x=y \cdot y^{-1} x \in$ $\left(\bigcap_{i \in I} R_{i}\right)\left(\prod_{j \in J} R_{j}\right)$. Equation (7) holds.

For the other direction, we suppose (7) is true. $\overline{\bigcap_{i \in I} R_{i}} \subseteq \overline{R_{i}}$ for any $i$, so $\overline{\bigcap_{i \in I} R_{i}} \subseteq$ $\bigcap_{i \in I} \bar{R}_{i}$. Now for any $\bar{x} \in \bigcap_{i \in I} \overline{R_{i}}, \bar{x} \in \overline{R_{i}}$ for any $i$. Then $x \in \pi^{-1}(\bar{x}) \subset$ $\bigcap_{i \in I}\left(R_{i}\left(\prod_{j \in J} R_{j}\right)\right)=\left(\bigcap_{i \in I} R_{i}\right)\left(\prod_{j \in J} R_{j}\right)$, where the last equality follows from (7). So $\pi(x) \in \overline{\bigcap_{i \in I} R_{i}}$. Equation (10) holds.

Proof of Lemma 2.1 For the cases that $n \leq 3$, the proofs are trivial. So without loss of generality, we assume $n>3, I=\{1, \ldots, n-2\}$ and $J=\{n-1\}$. For other cases, the proofs are similar.

Suppose that $\bar{G}=G\left(L_{n}\right) / \prod_{j \in J} R_{j}$. We notice that $\bar{G}$ is a free group generated by $\left\{\overline{\alpha_{i}}=\alpha_{i} \mid i \notin J\right\}$ since $J$ is nonempty. So $\bar{G}=F\left(\overline{\alpha_{1}}, \ldots, \overline{\alpha_{n-2}}, \overline{\alpha_{n}}\right)=$ $F\left(\alpha_{1}, \ldots, \alpha_{n-2}, \alpha_{n}\right)$. There is a group homomorphism

$$
F\left(\overline{\alpha_{1}}, \ldots, \overline{\alpha_{n-2}}\right) \rightarrow F\left(\overline{\alpha_{1}}, \ldots, \overline{\alpha_{i-1}}, \overline{\alpha_{i+1}}, \ldots \overline{\alpha_{n-2}}\right),
$$

so that $\overline{R_{i}}=\overline{\left\langle\alpha_{i}\right\rangle}$ is the kernel. By [1, Lemma 5.1], we have $\overline{R_{1}} \cap \cdots \cap \overline{R_{n-2}}=$ $\left[\overline{R_{1}}, \ldots, \overline{R_{n-2}}\right]_{S}$. We also notice that $\left[R_{1}, \ldots, R_{n-2}\right]_{S} \leq \bigcap_{k=1}^{n-2} R_{k}$. Now, if we think of the quotient map

$$
G\left(L_{n}\right) \stackrel{q}{\longrightarrow} \bar{G}
$$

we have

$$
\begin{aligned}
\bigcap_{k \in I} R_{k} & =q\left(\bigcap_{k \in I} R_{k}\right) \geq q\left(\left[R_{1}, R_{2}, \ldots, R_{n-2}\right]_{S}\right) \\
& =\left[\overline{R_{1}}, \overline{R_{2}}, \ldots, \overline{R_{n-2}}\right]_{S}=\bigcap_{k \in I} \overline{R_{k}} .
\end{aligned}
$$

The other direction is obvious.

The group $T\left(R_{1}, R_{2}, \ldots, R_{m}\right)$ in [10, Theorem 1$]$ is generated by symbols

$$
a \otimes_{A, B} b,
$$


where $A \sqcup B=\{1,2, \ldots, m\}, a \in R_{A}, b \in R_{B}$ (here for any $I \subset\{1,2, \ldots, m\}, R_{I}=$ $\left.\bigcap_{i \in I} R_{i}\right)$, and it satisfies the relations

$$
\begin{gathered}
a \otimes_{A, B} b=\left(b \otimes_{B, A} a\right)^{-1}, \\
a a^{\prime} \otimes_{A, B} b=\left({ }^{a} a^{\prime} \otimes_{A, B}{ }^{a} b\right)\left(a \otimes_{A, B} b\right), \\
\left({ }^{u}\left[u^{-1}, v\right] \otimes_{U \cup V, W}{ }^{u} w\right)\left({ }^{w}\left[w^{-1}, u\right] \otimes_{W \cup U, V}{ }^{w} v\right)\left({ }^{v}\left[v^{-1}, w\right] \otimes_{V \cup W, U}{ }^{v} u\right)=1, \\
\left(a \otimes_{A, B} b\right)\left(a^{\prime} \otimes_{A^{\prime}, B^{\prime}} b^{\prime}\right)\left(a \otimes_{A, B} b\right)^{-1}={ }^{[a, b]} a^{\prime} \otimes_{A^{\prime}, B^{\prime}}{ }^{[a, b]} b^{\prime}
\end{gathered}
$$

for $A \sqcup B=A^{\prime} \sqcup B^{\prime}=\{1,2, \ldots, m\}, a \in N_{A}, a^{\prime} \in N_{A^{\prime}}, b \in N_{B}, b^{\prime} \in N_{B^{\prime}}, U \sqcup V \sqcup W=$ $\{1,2, \ldots, m\}, u \in N_{U}, v \in N_{V}, w \in N_{W}$. Here we define ${ }^{x} y=x y x^{-1}$.

For an $m$-tuple $\Delta \in\{0,1\}^{m}$, there is an $m$-cube of spaces $B=B\left(G, R_{1}, \ldots, R_{m}\right)$ : $\{0,1\}^{m} \rightarrow$ (spaces) for which

$$
B_{\Delta}=B\left(\frac{G}{\prod_{i \in \Delta} R_{i}}\right)
$$

is the classifying space of the quotient group $G / \prod_{i \in \Delta} R_{i}$. Here $i \in \Delta$ means the $i^{\text {th }}$ coordinate of $\Delta$ equals 1 . This idea can be easily extended to an $m$-tuple $\Delta \in$ $\{-1,0,1\}^{m}$. It was shown $[4 ; 21]$ that each $m$-cube of spaces corresponds to an $m$-cube of fibrations $F:\{-1,0,1\}^{m} \rightarrow$ (spaces). The homomorphism

$$
T\left(R_{1}, \ldots, R_{m}\right) \rightarrow \pi_{1}\left(F_{-1,-1, \ldots,-1,0}\right) \leq G, \quad x \otimes y \mapsto[x, y]
$$

has a crossed module structure $\partial: T\left(R_{1}, \ldots, R_{m}\right) \rightarrow G, x \otimes y \mapsto[x, y]$ such that for any $g \in G,{ }^{g}(x \otimes y)=\left({ }^{g} x \otimes{ }^{g} y\right)$.

\section{Homotopy type of $X\left(L_{n}\right)^{m}$}

In this section we will study the homotopy type of the classifying space $X\left(L_{n}\right)^{m}$. When $n=2$, it's immediate to see that $X\left(L_{2}\right)^{2} \simeq S^{3}$, because $S^{3}$ is the push-out of $K(\pi, 1)$-spaces $S^{3} \backslash d_{2} L_{2} \leftarrow S^{3} \backslash L_{2} \rightarrow S^{3} \backslash d_{1} L_{2}$. We choose the case $n=4$ as an experimental example; the case $n=3$ is similar. The more general cases will be studied inductively.

\section{A The experimental example $n=4$}

To get $X\left(L_{4}\right)^{2}$ we study the cubical diagram

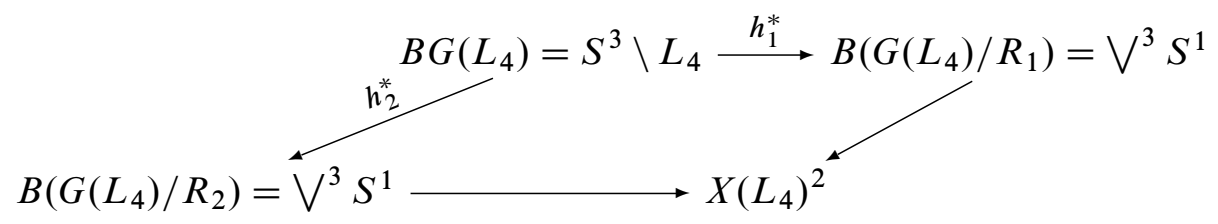


where $h_{1}^{*}$ and $h_{2}^{*}$ are induced from the group homomorphisms $h_{1}$ and $h_{2}$.

In order to get the homotopy type of the push-out $X\left(L_{4}\right)^{2}$ we need to look into the structure of $B\left(G\left(L_{4}\right) / R_{1}\right)$ and $B\left(G\left(L_{4}\right) / R_{2}\right)$. Let $d_{1} L_{4}=\left\{l_{2}, l_{3}, l_{4}\right\}$ be the 3 -link obtained by deleting the first component of $L_{4}$. The link complement $S^{3} \backslash d_{1} L_{4}$ has same the homotopy type as $\left(\bigvee^{3} S^{1}\right) \vee\left(\bigvee^{2} S^{2}\right)$ since $d_{1} L_{4}$ is splittable; we refer to [11, Proposition 2.2] for more details. The 2 -sphere $S_{i}^{2}, i=2,3$, is a separating sphere which separates $l_{i}$ from the other components. Then the classifying space $B\left(G\left(L_{4}\right) / R_{1}\right)$ is obtained by filling in the 2-spheres from $\left(\bigvee^{3} S^{1}\right) \vee\left(\bigvee^{2} S^{2}\right)$ with 3balls, ie $B\left(G\left(L_{4}\right) / R_{1}\right)=\left(S^{3} \backslash d_{1} L_{4}\right) \cup\left(\bigcup^{2} D^{3}\right)=\left(\left(\bigvee^{3} S^{1}\right) \vee\left(\bigvee^{2} S^{2}\right) \cup\left(\bigcup^{2} D^{3}\right)=\right.$ $\bigvee^{3} S^{1}$. The left side of Figure 1 will show us the idea more clearly.
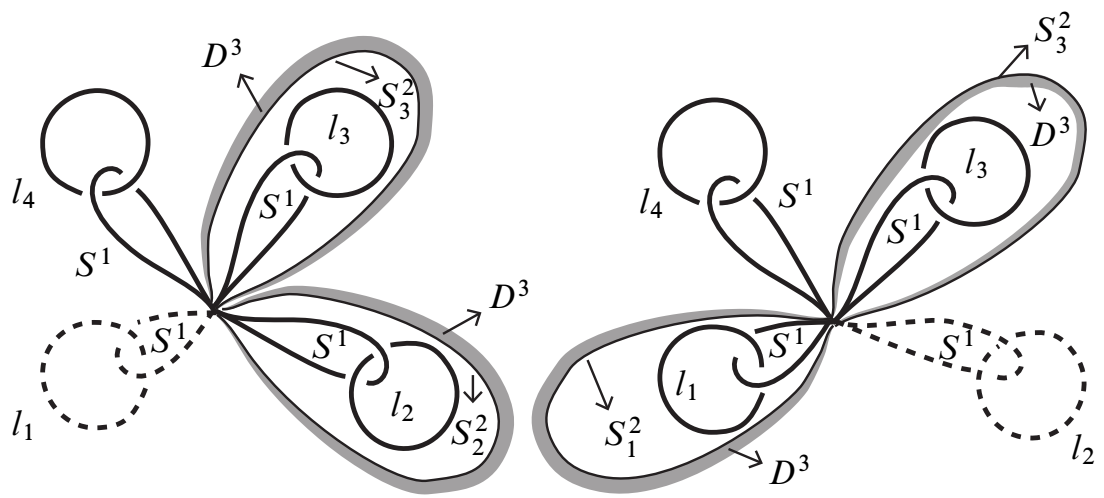

Figure 1: The classifying space $B\left(G\left(L_{4}\right) / R_{1}\right)$ is obtained by filling in the 2-spheres from $\left(\bigvee^{3} S^{1}\right) \vee\left(\bigvee^{2} S^{2}\right)$ with 3 -balls (left); $B\left(G\left(L_{4}\right) / R_{2}\right)$ is obtained similarly (right).

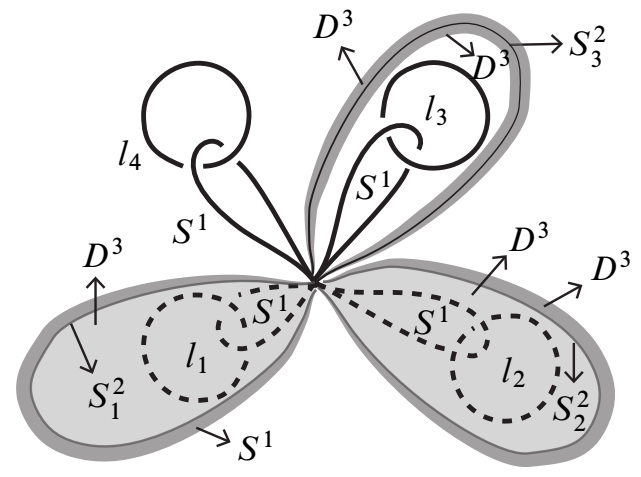

Figure 2: $X\left(L_{4}\right)^{2}$ is homotopic to $\left(\bigvee^{2} S^{1}\right) \vee\left(\bigvee^{3} S^{3}\right)$.

By the same argument, we can determine the homotopy type and structure of the space $B\left(G\left(L_{4}\right) / R_{2}\right)$, which is illustrated on the right in Figure 1. 
By definition, the homotopy push-out $X\left(L_{4}\right)^{2}$ is homotopic to $\left(\bigvee^{2} S^{1}\right) \vee\left(\bigvee^{3} S^{3}\right)$ as indicated in Figure 2.

Actually, the space $X\left(L_{4}\right)^{2}$ is obtained from the disjoint union of $B\left(G\left(L_{4}\right) / R_{1}\right)=$ $\left(\left(\bigvee^{3} S^{1}\right) \vee\left(\bigvee^{2} S^{2}\right)\right) \cup\left(\bigcup^{2} D^{3}\right), B\left(G\left(L_{4}\right) / R_{2}\right)=\left(\left(\bigvee^{3} S^{1}\right) \vee\left(\bigvee^{2} S^{2}\right)\right) \cup\left(\bigcup^{2} D^{3}\right)$ and $\left(S^{3} \backslash L_{4}\right) \times[0,1]$ by identifying $\left(S^{3} \backslash L_{4}\right) \times\{0\}$ (respectively $\left.\left(S^{3} \backslash L_{4}\right) \times\{1\}\right)$ with $h_{1}^{*}\left(S^{3} \backslash L_{4}\right) \subset B\left(G\left(L_{4}\right) / R_{1}\right)$ (respectively $\left.h_{2}^{*}\left(S^{3} \backslash L_{4}\right) \subset B\left(G\left(L_{4}\right) / R_{2}\right)\right)$. Notice that now the component $l_{1}$ (respectively $l_{2}$ ) is filled in, so one side of the 2 -sphere $S_{1}^{2}$ (respectively $\left.S_{2}^{2}\right)$ is $\left(D^{3} \backslash l_{1}\right) \cup l_{1}=D^{3}$ (respectively $\left(D^{3} \backslash l_{2}\right) \cup l_{2}=D^{3}$ ) while the other side is also a 3 -ball, ie there is a 3 -sphere.

For $m=3$, we refer to the cubical diagram in Figure 3.

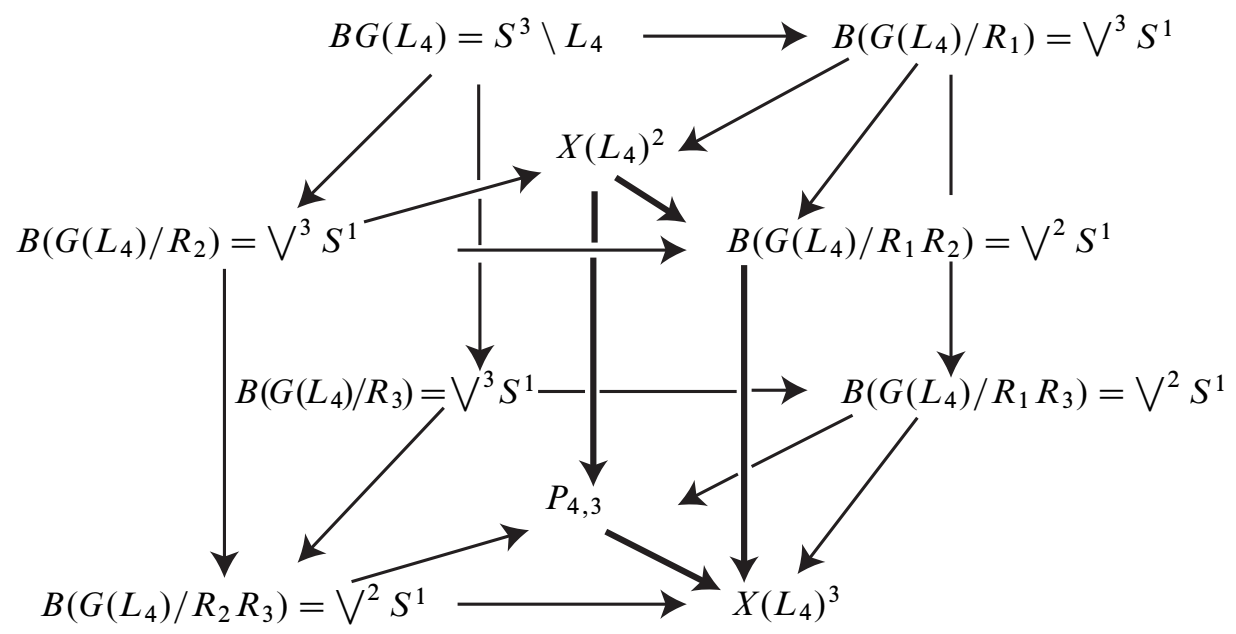

Figure 3

There is a natural inclusion from $X\left(L_{4}\right)^{2}=\left(\bigvee^{2} S^{1}\right) \vee\left(\bigvee^{3} S^{3}\right)$ to $B\left(G\left(L_{4}\right) / R_{1} R_{2}\right)=$ $\bigvee^{2} S^{1}$. Actually the space $B\left(G\left(L_{4}\right) / R_{1} R_{2}\right)$ can be thought of as obtained from $X\left(L_{4}\right)^{2}$ by filling in the three $S^{3}$ components by $4-$ balls, ie $B\left(G\left(L_{4}\right) / R_{1} R_{2}\right)=$ $X\left(L_{4}\right)^{2} \cup\left(\bigcup^{3} D^{4}\right)=\left(\left(\bigvee^{2} S^{1}\right) \vee\left(\bigvee^{3} S^{3}\right)\right) \cup\left(\bigcup^{3} D^{4}\right)=\bigvee^{2} S^{1}$.

Now, let's study the bottom level of the cubical diagram. We have $B\left(G\left(L_{4}\right) / R_{3}\right)=$ $\left(S^{3} \backslash d_{3} L_{4}\right) \cup\left(\bigcup^{2} D^{3}\right)=\bigvee^{3} S^{1} . B\left(G\left(L_{4}\right) / R_{1} R_{3}\right)$ (respectively $B\left(G\left(L_{4}\right) / R_{2} R_{3}\right)$ ) can be thought of as obtained from the space $B\left(G\left(L_{4}\right) / R_{3}\right)$ by filling in the $l_{1}$ (respectively $l_{2}$ ) component and then capping off the resulting $S^{3}$ boundary by a $D^{4}$, ie $B\left(G\left(L_{4}\right) / R_{1} R_{3}\right)=\left(S^{3} \backslash d_{3} L_{4}\right) \cup\left(\cup^{2} D^{3}\right) \cup l_{1} \cup D^{4}=\bigvee^{2} S^{1}$ (respectively $\left.B\left(G\left(L_{4}\right) / R_{2} R_{3}\right)=\left(S^{3} \backslash d_{3} L_{4}\right) \cup\left(\cup^{2} D^{3}\right) \cup l_{2} \cup D^{4}=\bigvee^{2} S^{1}\right)$, as shown in Figure 4. 
By the definition of homotopy push-out, the space $P_{4,3}$ is determined by the diagram

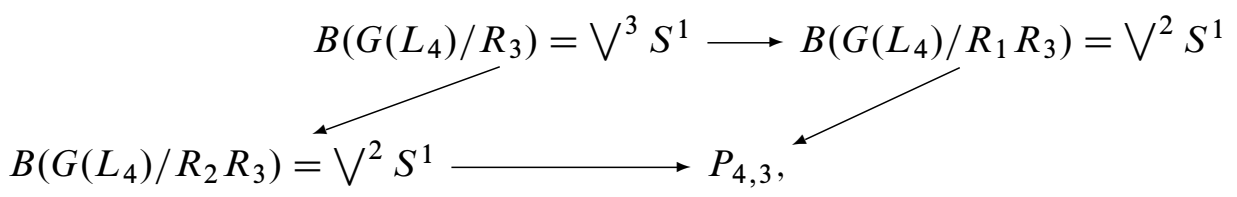

and $P_{4,3}$ is isomorphic to $S^{1}$; see Figure 5. On the other hand, $P_{4,3}$ can also be thought of as obtained from $X\left(L_{4}\right)^{2}$ by removing the $l_{3}$ component and capping off all three $S^{3}$ components by $4-$ balls; see Figure 6 .

Then the space $X\left(L_{4}\right)^{3}$ is determined by the diagram

$$
P_{4,3}=S^{1} \longrightarrow X\left(L_{4}\right)^{2} \longrightarrow B\left(G\left(L_{4}\right)^{3},\right.
$$

where the space $B\left(G\left(L_{4}\right) / R_{1} R_{2}\right)$ is obtained from $X\left(L_{4}\right)^{2}$ by filling in the three $S^{3}$ components by 4 -balls, as shown in Figure 7. We conclude that $X\left(L_{4}\right)^{3}=$ $S^{1} \vee\left(\bigvee^{3} S^{4}\right)$.

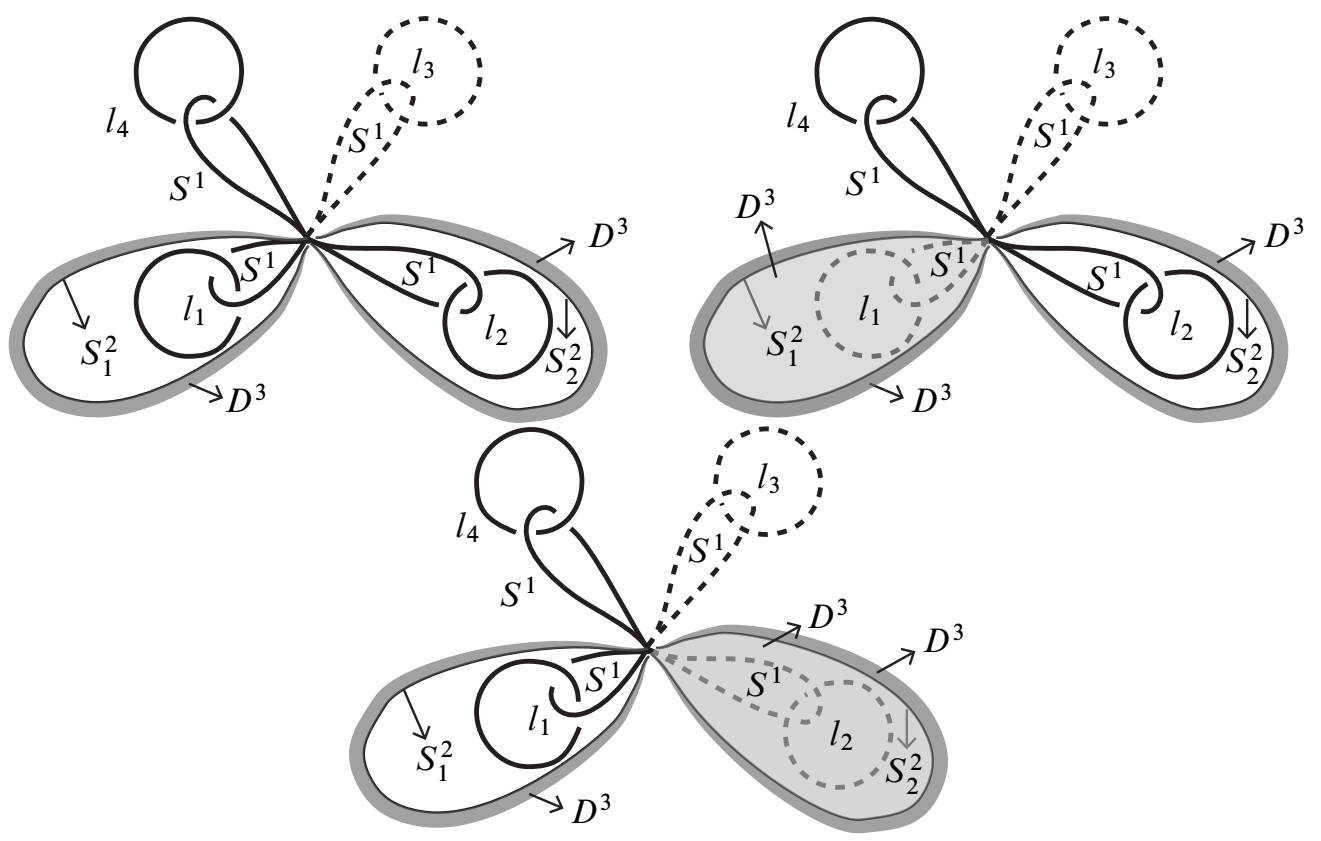

Figure 4: The classifying spaces $B\left(G\left(L_{4}\right) / R_{3}, B\left(G\left(L_{4}\right) / R_{1} R_{3}\right.\right.$, and $B\left(G\left(L_{4}\right) / R_{2} R_{3}\right.$, respectively 
The last case left is $X\left(L_{4}\right)^{4}$, which is determined by the following diagram:

$$
\begin{aligned}
& X\left(L_{4}\right)^{3}=S^{1} \vee\left(\bigvee^{3} S^{4}\right) \longrightarrow B\left(G\left(L_{4}\right) / R_{1} R_{2} R_{3}\right)=X\left(L_{4}\right)^{3} \cup\left(\bigcup^{3} D^{5}\right)=S^{1} \\
& P_{4,4} \longrightarrow X\left(L_{4}\right)^{4} \text {, }
\end{aligned}
$$

where the space $B\left(G\left(L_{4}\right) / R_{1} R_{2} R_{3}\right)$ is obtained from $X\left(L_{4}\right)^{3}$ by capping off the $S^{4}$ components by 5 -balls, and the space $P_{4,4}$ is obtained by removing the $l_{4}$ component

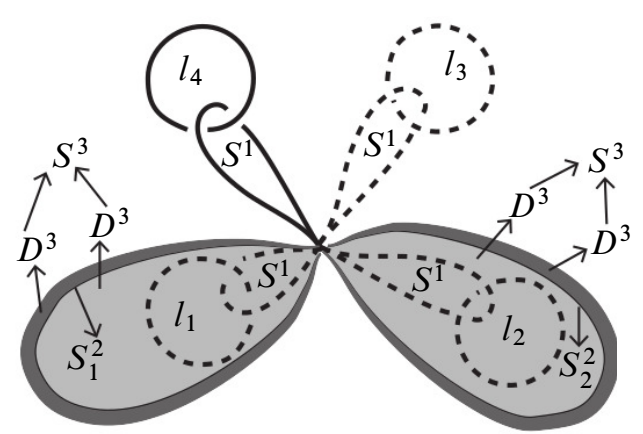

Figure 5: The pushout $P_{4,3}$ is isomorphic to $S^{1}$.

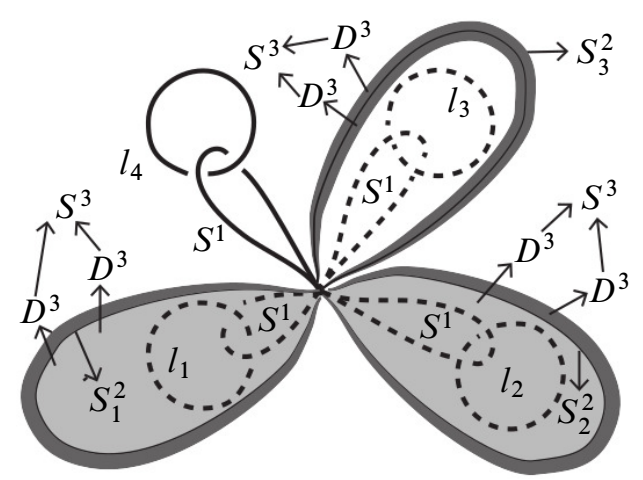

Figure 6: On the other hand, $P_{4,3}$ can be thought of as obtained from $X\left(L_{4}\right)^{2}$ by removing the $l_{3}$ component and capping off all the three $S^{3}$ components by $D^{4}$ 's.

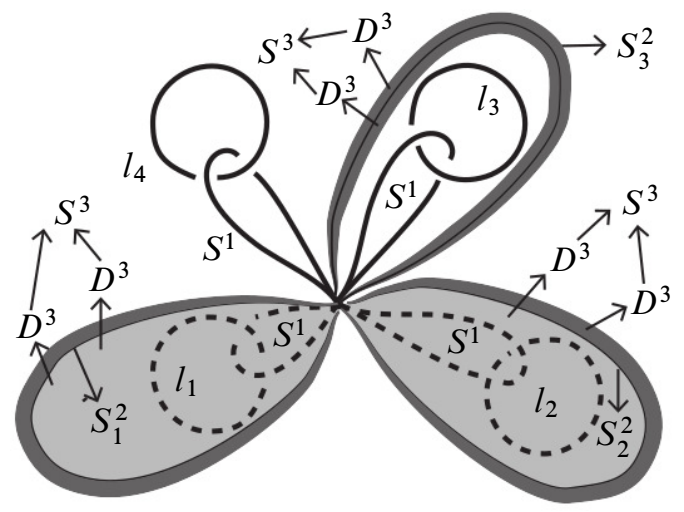

Figure 7: The space $B\left(G\left(L_{4}\right) / R_{1} R_{2}\right)$ is obtained from $X\left(L_{4}\right)^{2}$ by filling in the three $S^{3}$ components by $D^{4}$ 's. 
from $X\left(L_{4}\right)^{3}$ and capping off the $S^{4}$ components by 5 -balls, ie $P_{4,4}$ is isomorphic to a point. So $X\left(L_{4}\right)^{4}=\bigvee^{3} S^{5}$.

\section{B The general cases}

Based on the experimental example we studied in detail in the previous section, we'll sketch the procedure of finding the homotopy types of the homotopy colimits very roughly in this section.

For any $n>4$ and $2 \leq m \leq n$, we want to show that

$$
X\left(L_{n}\right)^{m}=\left(\bigvee^{n-m} S^{1}\right) \vee\left(\bigvee^{n-1} S^{m+1}\right)
$$

noticing that for $n=2,3,4$ this equality holds. Now, for any $n>4$, we assume that the spaces $X\left(L_{n}\right)^{k}, 2 \leq k \leq m-1$ satisfy this equation. By induction, we notice that $X\left(L_{n}\right)^{m}$ is determined by the diagram

$$
\begin{aligned}
X\left(L_{n}\right)^{m-1}= & \left(\bigvee^{n-m+1} S^{1}\right) \\
& \vee\left(\bigvee^{n-1} S^{m}\right)
\end{aligned} \quad \begin{aligned}
B\left(G\left(L_{n}\right) /\right. & \left.R_{1} \cdots R_{m-1}\right) \\
& =X\left(L_{n}\right)^{m-1} \cup\left(\bigcup^{n-1} D^{m+1}\right) \\
& =\bigvee^{n-m+1} S^{1}
\end{aligned}
$$

where $B\left(G\left(L_{n}\right) / R_{1} \cdots R_{m-1}\right)$ can be thought of as obtained from $X\left(L_{n}\right)^{m-1}$ by filling in the $S^{m}$ components, and $P_{n, m}$ is obtained from $X\left(L_{n}\right)^{m-1}$ by removing the $l_{m}$ component and capping off all the sphere components with solid balls. Then $P_{n, m}=\bigvee^{n-m} S^{1}$, and the colimit $X\left(L_{n}\right)^{m}=\left(\bigvee^{n-m} S^{1}\right) \vee\left(\bigvee^{n-1} S^{m+1}\right)$.

\section{Proofs of theorems}

In this section, we first study the $n^{\text {th }}$ and $(n+1)^{\text {st }}$ homotopy groups of the homotopy colimits, then the proofs of our theorems are just corollaries.

\section{A The homotopy groups of the homotopy colimits}

We have already shown that $X\left(L_{n}\right)^{m}=\left(\bigvee^{n-m} S^{1}\right) \vee\left(\bigvee^{n-1} S^{m+1}\right)$. 
For the case $m=n$, the homotopy groups of $X\left(L_{n}\right)^{n}$ can be obtained by applying the Hurewicz theorem. In fact, the homology groups of $X\left(L_{n}\right)^{n}$ satisfy the equalities

$$
\begin{gathered}
H_{n}\left(X\left(L_{n}\right)^{n}\right)=H_{n}\left(\bigvee^{n-1} S^{n+1}\right)=\bigoplus^{n-1} H_{n}\left(S^{n+1}\right)=0 \\
H_{n+1}\left(X\left(L_{n}\right)^{n}\right)=H_{n+1}\left(\bigvee^{n-1} S^{n+1}\right)=\bigoplus^{n-1} H_{n+1}\left(S^{n+1}\right)=\bigoplus^{n-1} \mathbb{Z} .
\end{gathered}
$$

So by the Hurewicz theorem, $\pi_{n}\left(X\left(L_{n}\right)^{n}\right)=0$ and $\pi_{n+1}\left(X\left(L_{n}\right)^{n}\right)=\bigoplus^{n-1} \mathbb{Z}$.

For the case $m<n, X\left(L_{n}\right)^{m}=\left(\bigvee^{n-m} S^{1}\right) \vee\left(\bigvee^{n-1} S^{m+1}\right)$. We consider the universal covering space $\tilde{X}$ of $X\left(L_{n}\right)^{m}$, which is an infinite tree with every vertex of valency $2(n-m)$, and with $n-1(m+1)-$ spheres attached to each vertex. So we obtain

$$
\begin{gathered}
\pi_{m}\left(X\left(L_{n}\right)^{m}\right)=\pi_{m}(\tilde{X})=0 \\
\pi_{m+1}\left(X\left(L_{n}\right)^{m}\right)=\pi_{m+1}(\tilde{X})=\bigoplus \mathbb{Z} .
\end{gathered}
$$

\section{B Proof of Theorem 1.1}

According to (8),

$$
0=\pi_{m}\left(X\left(L_{n}\right)^{m}\right) \cong \frac{R_{1} \cap \cdots \cap R_{m}}{\prod_{I \cup J=\{1,2, \ldots, m\}, I \cap J=\varnothing}\left[\bigcap_{i \in I} R_{i}, \bigcap_{j \in J} R_{j}\right]}
$$

for all $m$, ie $R_{1} \cap \cdots \cap R_{m}=\prod_{I \cup J=\{1,2, \ldots, m\}, I \cap J=\varnothing}\left[\bigcap_{i \in I} R_{i}, \bigcap_{j \in J} R_{j}\right]$. Obviously, $\left[R_{1}, \ldots, R_{m}\right]_{S} \leq R_{1} \cap \cdots \cap R_{m}$. The following lemma will conclude the proof.

Lemma 4.1 Based on the assumptions of Theorem 1.1,

$$
\left[R_{1}, \ldots, R_{m}\right]_{S} \geq \prod_{I \cup J=\{1,2, \ldots, m\}, I \cap J=\varnothing}\left[\bigcap_{i \in I} R_{i}, \bigcap_{j \in J} R_{j}\right]=R_{1} \cap \cdots \cap R_{m} .
$$

Proof According to $\left[28\right.$, Corollary 3.5], $\bigcap_{i \in I} R_{i}=\left[\left[R_{i_{1}}, \ldots, R_{i_{|I|}}\right]\right]$ and $\bigcap_{j \in J} R_{j}=$ $\left[\left[R_{j_{1}}, \ldots, R_{\left.j_{|J|}\right]}\right]\right.$, so

$$
\prod_{I \cup J=\{1,2, \ldots, m\}, I \cap J=\varnothing}\left[\bigcap_{i \in I} R_{i}, \bigcap_{j \in J} R_{j}\right] \leq\left[\left[R_{1}, \ldots, R_{m}\right]\right]=\left[R_{1}, \ldots, R_{m}\right]_{S} .
$$




\section{C Proof of Theorem 1.2}

According to (9),

$\operatorname{ker}\left(\partial: T\left(R_{1}, \ldots, R_{m}\right) \rightarrow G\left(L_{n}\right)\right)=\pi_{m+1}\left(X\left(L_{n}\right)^{m}\right)= \begin{cases}\bigoplus^{n-1} \mathbb{Z} & \text { when } m=n \\ \bigoplus^{\infty} \mathbb{Z} & \text { when } m<n .\end{cases}$

For all disjoint subsets $A, B \subset\{1,2, \ldots, m\}$ and any $x \in R_{A}, y \in R_{B}$, we have $\partial(x \otimes y)=[x, y] \in\left[\bigcap_{i \in A} R_{i}, \bigcap_{j \in B} R_{j}\right]$. By the arbitrariness of $A$ and $B$, we claim that $\operatorname{Im}(\partial)=\prod_{I \cup J=\{1,2, \ldots, m\}, I \cap J=\varnothing}\left[\bigcap_{i \in I} R_{i}, \bigcap_{j \in J} R_{j}\right]$. Further, we notice that there is an exact sequence

$$
\begin{aligned}
\operatorname{ker}(\partial)=\pi_{m+1}\left(X\left(L_{n}\right)^{m}\right) & \longrightarrow T\left(R_{1}, R_{2}, \ldots, R_{m}\right) \\
& \rightarrow \operatorname{Im}(\partial)=\prod_{I \cup J=\{1,2, \ldots, m\}, I \cap J=\varnothing}\left[\bigcap_{i \in I} R_{i}, \bigcap_{j \in J} R_{j}\right] \\
& \longmapsto \bigcap_{i=1}^{m} R_{i} \rightarrow \operatorname{Coker}(\partial)=\pi_{m}\left(X\left(L_{n}\right)^{m}\right)=0 .
\end{aligned}
$$

This concludes the proof.

\section{References}

[1] V G Bardakov, R Mikhailov, V V Vershinin, J Wu, Brunnian braids on surfaces, Algebr. Geom. Topol. 12 (2012) 1607-1648 MR2966697

[2] A J Berrick, F R Cohen, Y L Wong, J Wu, Configurations, braids, and homotopy groups, J. Amer. Math. Soc. 19 (2006) 265-326 MR2188127

[3] W A Bogley, J H C Whitehead's asphericity question, from "Two dimensional homotopy and combinatorial group theory" (C Hog-Angeloni, W Metzler, editors), London Math. Soc. Lecture Note Ser. 197, Cambridge Univ. Press, Cambridge (1993) 309-334 MR1279184

[4] R Brown, J-L Loday, Van Kampen theorems for diagrams of spaces, Topology 26 (1987) 311-335 MR899052

[5] H Brunn, Üeber Verkettung, Sitzungberichte der Bayerischer Akad. Wiss. Math.-Phys. 22 (1892) 77-99

[6] K S Chichak, S J Cantrill, A R Pease, S-H Chiu, G W V Cave, J L Atwood, J F Stoddart, Molecular Borromean Rings, Science 304 (2004) 1308-1312

[7] F R Cohen, J Wu, On braid groups and homotopy groups, from "Groups, homotopy and configuration spaces" (N Iwase, T Kohno, R Levi, D Tamaki, J Wu, editors), Geom. Topol. Monogr. 13 (2008) 169-193 MR2508205 
[8] F R Cohen, J Wu, Artin's braid groups, free groups, and the loop space of the 2-sphere, Q. J. Math. 62 (2011) 891-921 MR2853222

[9] H Debrunner, Links of Brunnian type, Duke Math. J. 28 (1961) 17-23 MR0137106

[10] G Ellis, R Mikhailov, A colimit of classifying spaces, Adv. Math. 223 (2010) 20972113 MR2601009

[11] F Fang, F Lei, J Wu, The symmetric commutator homology of link towers and homotopy groups of 3-manifolds, Commun. Math. Stat. 3 (2015) 497-526 MR3432218

[12] G G Gurzo, The group of smooth braids, from "16th All-Union Algebra Conference", Abstract II, Leningrad (1981) 39-40

[13] K Habiro, Claspers and finite type invariants of links, Geom. Topol. 4 (2000) 1-83 MR1735632

[14] K Habiro, Brunnian links, claspers and Goussarov-Vassiliev finite type invariants, Math. Proc. Cambridge Philos. Soc. 142 (2007) 459-468 MR2329695

[15] K Habiro, J-B Meilhan, On the Kontsevich integral of Brunnian links, Algebr. Geom. Topol. 6 (2006) 1399-1412 MR2253452

[16] K Habiro, J-B Meilhan, Finite type invariants and Milnor invariants for Brunnian links, Internat. J. Math. 19 (2008) 747-766 MR2431635

[17] D L Johnson, Towards a characterization of smooth braids, Math. Proc. Cambridge Philos. Soc. 92 (1982) 425-427 MR677467

[18] H Levinson, Decomposable braids and linkages, Trans. Amer. Math. Soc. 178 (1973) 111-126 MR0324684

[19] H Levinson, Decomposable braids as subgroups of braid groups, Trans. Amer. Math. Soc. 202 (1975) 51-55 MR0362287

[20] J Y Li, J Wu, On symmetric commutator subgroups, braids, links and homotopy groups, Trans. Amer. Math. Soc. 363 (2011) 3829-3852 MR2775829

[21] J-L Loday, Spaces with finitely many nontrivial homotopy groups, J. Pure Appl. Algebra 24 (1982) 179-202 MR651845

[22] W Magnus, A Karrass, D Solitar, Combinatorial group theory: Presentations of groups in terms of generators and relations, Interscience, New York (1966) MR0207802

[23] B Mangum, T Stanford, Brunnian links are determined by their complements, Algebr. Geom. Topol. 1 (2001) 143-152 MR1823496

[24] J-B Meilhan, A Yasuhara, Whitehead double and Milnor invariants, Osaka J. Math. 48 (2011) 371-381 MR2831978

[25] J Milnor, Link groups, Ann. of Math. 59 (1954) 177-195 MR0071020

[26] H A Miyazawa, A Yasuhara, Classification of n-component Brunnian links up to $C_{n}$-move, Topology Appl. 153 (2006) 1643-1650 MR2227018 
[27] M Ozawa, On a genus of a closed surface containing a Brunnian link, Tokyo J. Math. 31 (2008) 347-349 MR2477877

[28] J Wu, Combinatorial descriptions of homotopy groups of certain spaces, Math. Proc. Cambridge Philos. Soc. 130 (2001) 489-513 MR1816806

School of Mathematical Sciences, Dalian University of Technology Dalian, 116024, China

Department of Mathematics, National University of Singapore 10 Lower Kent Ridge Road, Singapore 119076, Singapore Department of Mathematics, Harbin Institute of Technology Harbin, 150001, China

fclei@dlut.edu.cn, matwuj@nus.edu.sg, yuzhanghit@hit.edu.cn

Received: 8 April 2015 
